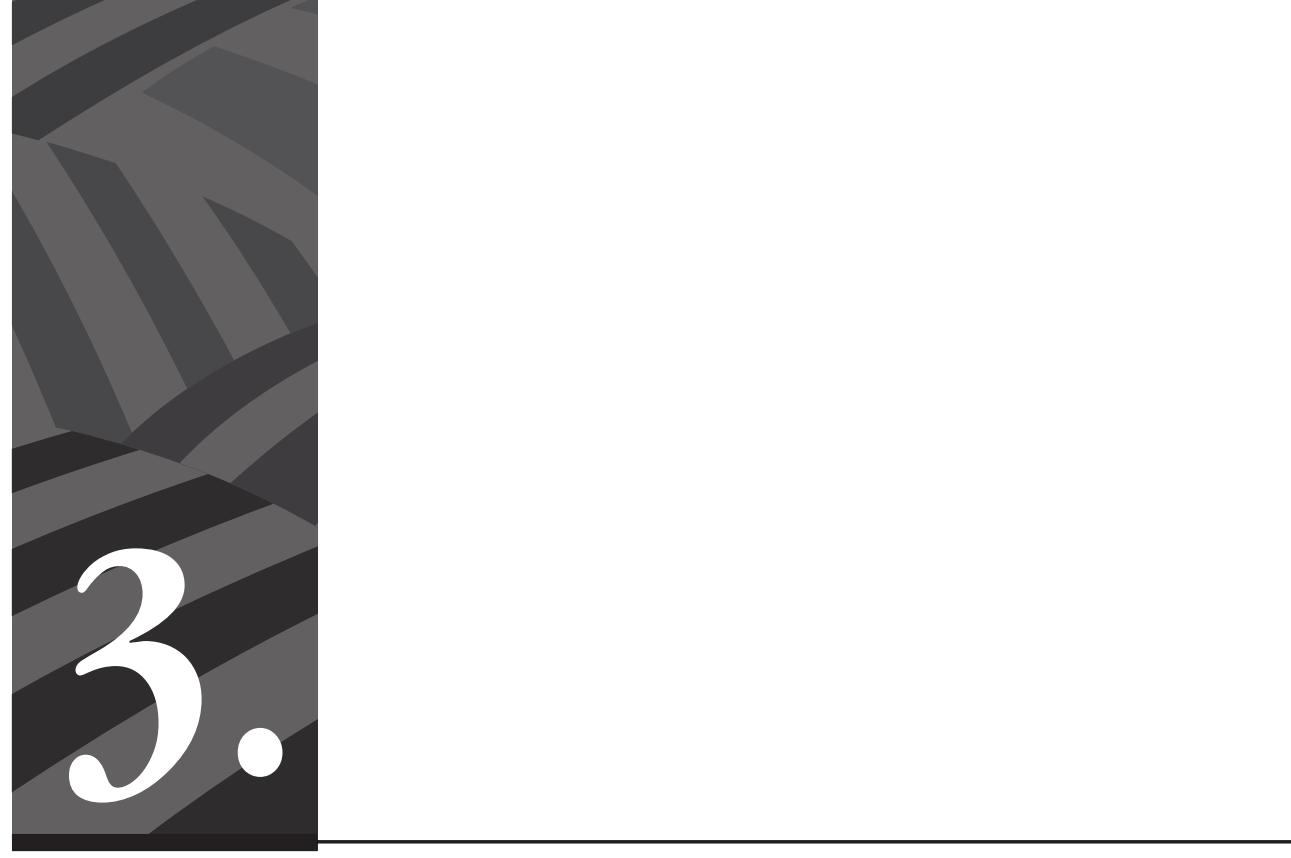

\title{
Sobre derechos humanos en la actividad empresarial en entornos multiculturales (e interculturales)
}




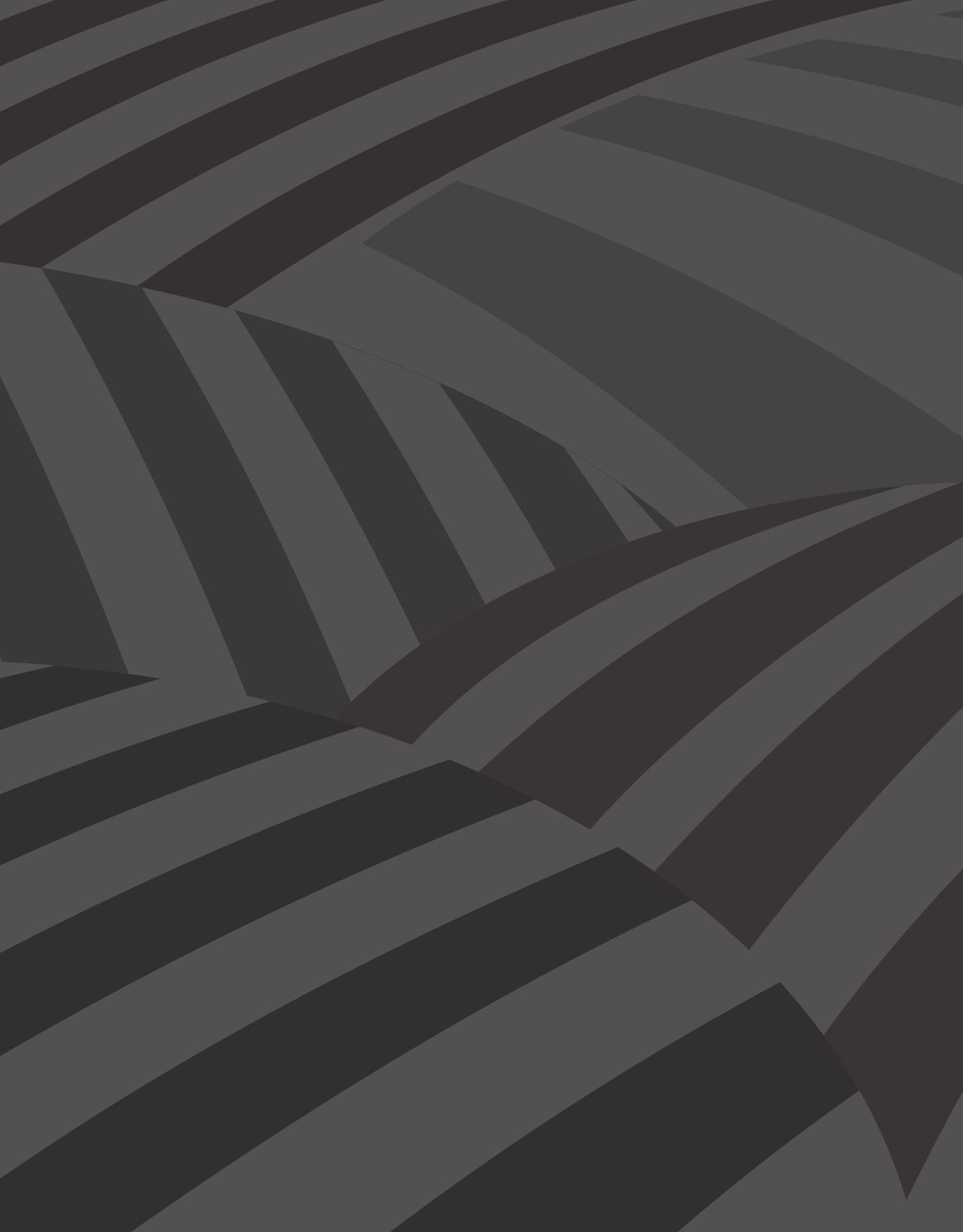




\section{Sobre derechos humanos en la actividad empresarial en entornos multiculturales (e interculturales)}

Por Luisa Fernanda Duque Muñoz*, Laura Álvarez Tobar** , María Jimena Fernández Merchán***

Resumen: El presente artículo sugiere una aproximación metodológica para aplicar el enfoque de derechos humanos a la actividad empresarial en entornos multiculturales (o interculturales), zonas con influencia del conflicto armado o donde exista una ausencia del Estado. Para ello se lleva a cabo una revisión de dos casos (María la Baja y Buenaventura), los cuales permiten examinar la relación entre empresas y derechos humanos, a la luz de los Principios Rectores sobre las empresas y los derechos humanos (PR), y su ejecución en Colombia. Se insiste en la importancia de continuar con el estudio de este tema y en que las empresas, en tanto actores con amplia injerencia en los territorios, den cumplimiento a estos principios.

Palabras clave: Empresas y derechos humanos, Principios Rectores de Empresa y Derechos Humanos, Plan Nacional de Acción, Multiculturalidad, Interculturalidad, Metodología.

* Investigadora del Instituto de Estudios Interculturales (IEI) de la Universidad Javeriana de Cali desde el año 2013. En la actualidad es abogada litigante. Magíster en Defensa de derechos humanos ante organismos, tribunales y cortes internacionales de la Universidad Santo Tomas.

** Investigadora en el Instituto de Estudios Interculturales de la Universidad Javeriana de Cali desde el año 2016. En la actualidad hace parte de la línea Sistemas económicos: derechos humanos, interculturalidad y sostenibilidad. Es politóloga con énfasis en gobernabilidad e integración al sistema internacional de la Universidad Javeriana de Cali.

*** Se desempeña como investigadora en el Instituto de Estudios Interculturales de la Universidad Javeriana de Cali en su línea Sistemas económicos: derechos humanos, interculturalidad y sostenibilidad desde marzo del 2018. Es politóloga con énfasis en gobernabilidad democrática e integración al sistema internacional de la Universidad Javeriana de Cali. 


\section{On human rights in the corporate action in multicultural (and pluricultural contexts)}

Abstract: This paper proposes a methodology for applying the human rights approach to business activity in multicultural (or intercultural) environments; areas that are under the influence of armed conflict; or where there is poor State presence. Through the examination of 2 case studies (María la Baja and Buenaventura) we examine the relationship between business and human rights, under the framework of the Guiding Principles on Human Rights and Business and their implementation in Colombia. We focus on the importance of addressing this issue and demanding one of the actors that has more influence in territories, such as corporations, to comply with these principles.

Keywords: Corporations and human rights, Guiding principles on business and human rights, National Action Plans, Multiculturality, Interculturality, Methodology.

Cómo citar este artículo: Duque Muñoz, Luisa Fernanda; Álvarez Tobar, Laura y Fernández Merchán, María Jimena (2019). Sobre derechos humanos en la actividad empresarial en entornos multiculturales (e interculturales). Revista Controversia, 212, 69-104.

Fecha de recepción: 30 de octubre de 2018

Fecha de aprobación: 2 de febrero de 2019

\section{Introducción}

$\mathrm{E}$

n el ámbito de los derechos humanos, un tema que hoy ha comenzado a tener mayor relevancia es la relación empresa-derechos humanos. La condición para abordar esta perspectiva no es fácil, teniendo en cuenta que aborda un cambio de paradigma. La manifestación inicial de que el Estado es el único responsable por las vulneraciones a estos derechos se ha modificado. Es así que no puede perderse de vista que si bien es el Estado el que debe promulgar su cumplimiento, así como el principal responsable de transgredirlos, todos los seres humanos debemos actuar acorde a los derechos humanos. En este sentido, en la actualidad con el amplio poder económico y político con el cual cuentan las empresas, es fundamental llegar a un consenso que permita establecer sus responsabilidades por las vulneraciones a estos derechos. 
Por este motivo, se han expedido normas voluntarias, siendo la principal de ellas los Principios Rectores sobre las empresas y los derechos humanos (en adelante PR).

Sin embargo, las normas que reglamentan esta relación -empresa y derechos humanos - se catalogan dentro de las normas voluntarias o soft law. Estas normas terminan adhiriéndose más a las condiciones sociales que aquellas de obligatorio cumplimiento o hard law, ya que las primeras tienen un sentido de costumbre y moral fuertemente marcado en la sociedad.

En este sentido, puede observarse cómo la sociedad civil ha intentado frenar prácticas empresariales que afectan los derechos humanos, tales como: la esclavitud moderna en empresas textiles en el mundo; prácticas de empresas de extracción de recursos no renovables, no sostenibles con el territorio y su entorno; proveedores con malas prácticas dentro de la cadena de valor (el caso más conocido es el de Nike); y la participación en conflictos armados. Todo ello en cumplimiento de normas correspondientes al soft law, que logran transformar las prácticas que atentan contra los derechos humanos.

El desarrollo de esta perspectiva de los derechos humanos debe contar con una metodología que permita su aplicación en entornos como el de Colombia, donde la multiculturalidad y la interculturalidad son características fundamentales del territorio. En Colombia confluyen diferentes actores, con diferentes culturas, cuyas prácticas y formas de relacionarse son distintas. Esto invita a pensar en prácticas industriales en los territorios que integren un enfoque de derechos humanos y empresa de una forma más efectiva; sobre todo donde el conflicto armado ha marcado unas condiciones especiales en esa forma de relacionamientos. Tampoco debe olvidarse que Colombia es un país en vías de desarrollo.

El presente artículo busca, en primera instancia, generar un interés superior en el ámbito de lo relacionado con derechos humanos y empresa. 
En segunda instancia, pretende realizar una aproximación metodológica para aplicar este enfoque de derechos humanos a la actividad empresarial en entornos multiculturales (o interculturales). En este punto se resalta la importancia de desarrollar la actividad empresarial sustentada en el respeto y la consideración de los derechos humanos. Esta metodología está pensada para ser aplicada a grandes empresas, tales como la agroindustria o puertos, pero, en definitiva, podría ser utilizada por cualquier empresa que se encuentre en un territorio que tenga como característica principal la diversidad cultural; presencia estatal precaria y territorios donde exista o hubiese existido conflicto armado.

\section{Desarrollo de los derechos humanos en la actividad empresarial}

Es importante iniciar este apartado con la siguiente pregunta ¿En qué momento se hacen responsables las empresas de las vulneraciones a los derechos humanos? Para dar respuesta a este interrogante, es pertinente tener en cuenta que las empresas pueden tener un gran poder de injerencia en el Estado, en diferentes ámbitos (políticos, sociales, económicos). En la época más reciente, estas han asumido funciones que antes cumplían directamente los Estados ${ }^{1}$.

Para los años noventa se profundizó especialmente en la Responsabilidad Social Empresarial (en adelante RSE) o Responsabilidad Social Corporativa para las multinacionales. Su definición no puede ser estática al igual que su esencia, De La Cuesta y Valor (2003) lo definen como un «conjunto de obligaciones y compromisos, legales y éticos, nacionales e internacionales con grupo de interés que se derivan de los impactos que la actividad y operaciones de las organizaciones producen en el ámbito social, laboral, medio ambiental y de los derechos humanos» (p. 7).

1 Como ejemplo podríamos citar el préstamo de servicios públicos por empresas privadas que antes estaba a cargo de empresas municipales o nacionales. 
De acuerdo con esta definición, se trataba, por lo tanto, de cumplir con los compromisos de los derechos humanos, pero no se tenía en el panorama la posibilidad de que las empresas fueran responsables por la vulneración de estos derechos. Esto tiene sentido por cuanto en el campo jurídico, los responsables de estos hechos eran los Estados. Sin embargo, desde comienzo de siglo se está cambiando el paradigma: los Estados no son los únicos responsables de las vulneraciones de los derechos humanos, dado que los cambios globales han generado esta transformación. En esta línea, Salmón et. al. (2016) manifiestan que

Las empresas y los derechos humanos no se encuentran distanciados. Por el contrario, somos testigos de un consenso creciente respecto al papel que juegan las empresas en el marco del respeto y garantía de estos derechos, tanto en su carácter individual como colectivo. Sin embargo, no suele quedar claro a qué nos estamos refiriendo cuando afirmamos que, en efecto, las empresas tienen algún tipo de obligación hacia los derechos humanos, que muchas veces se asocia únicamente a los Estados. En ese panorama, además, no solo hay que enfatizar el rol que cumplen las empresas en general, sino en especial aquellas denominadas multinacionales. La razón de esta especialidad es que las acciones de estas empresas impactan no solo en las condiciones de vida de las personas, sino también en la sociedad en su conjunto, así como en las economías, en el desarrollo, tanto de manera local como global. (p. 13)

Finalizando la primera década del 2000, se redefine la Responsabilidad Social Empresarial, partiendo de la necesidad de comprometer a las empresas con el cumplimiento de los derechos humanos y generar sanciones por las acciones que ejecuten en su contra, en razón a la injerencia que tienen en los territorios que ocupan. Es así como existen las normas ISO 26000 y el Pacto Global, que son voluntarias y ampliamente respetadas por las empresas, y contienen capítulos que desarrollan lo relacionado con el cumplimiento de los derechos humanos por parte de las empresas. 
En el mismo periodo surgen los PR asumidos por el Consejo de Derechos Humanos de la Organización de las Naciones Unidas (en adelante ONU), mediante la resolución 17/4 de 16 de junio del 2011, cuyo autor fue el profesor John Gerard Ruggie. Estos Principios cuentan con tres puntos fundamentales que son: proteger, respetar y remediar. Ruggie arguye que «los PR son un instrumento de derecho blando que prescribe estándares mínimos de conducta para todos los Estados y las empresas con respecto a los derechos humanos en su conjunto» (2016, p. 85). En este sentido, se afirma que los PR son las normas básicas que deberán regir a las empresas en el tema de derechos humanos, teniendo en cuenta que estas son un actor que suele operar en diferentes frentes en los Estados.

Estos Principios Rectores deben entenderse como un todo coherente y ser interpretados, individual y colectivamente, en términos de su objetivo de mejorar las normas y prácticas en relación con las empresas y los derechos humanos a fin de obtener resultados tangibles para las personas y las comunidades afectadas, y contribuir así también a una globalización socialmente sostenible. (ONU, 2011, p. 1)

La construcción de los PR, según manifiesta Ruggie (2014), no fue caprichosa. El contexto global ameritaba una transformación en el entendimiento de los derechos humanos y las repercusiones que sobre ellos tienen las empresas. Si bien en la década de los noventa se había avanzado en temas tales como medioambiente y ámbito social, aún existían carencias en puntos fundamentales en la RSE, en particular en lo relacionado con los derechos humanos. De ahí que, estos fueran los principales motivos para recurrir a unas normas que trascendieran la voluntariedad y se perfilaran por ser obligatorias a las empresas por cuanto se habían establecido denuncias en casos como: complicidad en conflictos armados y vulneraciones de derechos laborales que afectan la dignidad humana en las cadenas de valor (el caso más conocido al respecto es el de Nike). Así, estos correspondieron a la motivación de realizar un estudio amplio sobre cómo evitar la ocurrencia de estos hechos y la consecuente expedición de los PR. 
En la actualidad, después de la construcción de los PR, se ha continuado con el encargo de elaboración del tratado que lleva varios años discutiéndose; del 15 al 19 de octubre del año 2018 se debatió sobre el tema, y en la actualidad la sociedad civil está solicitando a diferentes Estados para que respalden el tratado, sin ninguna repercusión exitosa hasta el momento.

Para poder aplicar los PR por parte de las empresas, se generaron unas guías. Destacan principalmente el principio de debida diligencia, que hace referencia a estar atentos a todas las acciones que se realizan, para superar los hechos que pueden estar ocasionando vulneraciones a derechos humanos e impedir que nuevos hechos puedan dar lugar a estas vulneraciones por parte de las empresas. Estas guías hacen manifiesta la necesidad de tener consideración con la actividad empresarial en territorios que comprenda población con características para ser más vulnerables, como zonas de conflicto o poblaciones indígenas.

En Colombia, en el gobierno de Juan Manuel Santos, se asumió como política pública la generación del Plan de Acción Nacional (en adelante PNA, por su denominación en Colombia), acatando el llamado que hizo el grupo de trabajo de la ONU sobre empresa y derechos humanos en el informe del 2012 para el desarrollo de Planes de Acción Nacional (Cantú, 2017), esto con el fin de integrar como política pública los PR. Igualmente, durante el gobierno Santos, Colombia se incorporó en la Organización para la Cooperación y el Desarrollo Económico (OCDE), cuyo objetivo es coordinar las políticas económicas y sociales de los países que hacen parte. Allí se conformaron las Líneas Directrices de la OCDE para Empresas Multinacionales, «las cuales son recomendaciones dirigidas por los gobiernos a las empresas multinacionales [...] para promover una conducta empresarial responsable» (OCDE, 2013). Estas líneas fueron adoptadas a través del decreto 1400 del 2012, que contiene un apartado de derechos humanos. Igualmente, se configura el Punto Nacional de Contacto (PNC), en el cual se establece que una de sus funciones es atender solicitudes de incumplimiento de directrices. 
En relación con el PNA, se priorizaron tres frentes, considerados los más importantes a desarrollar en ese momento, a saber: minero-energético, agroindustria e infraestructura, en razón a que estos presentaban más oportunidades de trabajo. El PNA se estructura en los tres pilares establecidos en los PR: proteger, respetar y remediar.

Además, en el desarrollo del PNA se establecieron diferentes acciones para ejecutar cada uno de estos puntos:

En relación con el componente de proteger, que corresponde al Estado, definió:

- Coordinación interinstitucional.

- El Estado como actor económico.

- Participación efectiva de la sociedad civil.

- Orientación del Estado para el respeto a los derechos humanos en la actividad empresarial.

- Debida diligencia en derechos humanos.

- Cultura de derechos humanos y construcción de paz en el sector empresarial.

En lo que concierne al componente respetar, el cual es dado principalmente a la responsabilidad empresarial, estableció:

- Debida diligencia en el sector empresarial.

- Respeto a los derechos humanos como ventaja competitiva.

- Responsabilidad social empresarial y respeto por los derechos humanos.

Con respecto a remediar, consideró:

- Fortalecer los mecanismos judiciales y administrativos de acceso a remediación.

- Fortalecer los mecanismos no judiciales de remediación. 
En el último punto, con respecto a los mecanismos no judiciales, se entiende que el PNC es uno de estos, por lo que debe fortalecerse por parte del Estado para permitir la recepción de denuncias de las acciones cometidas por multinacionales.

Según el seguimiento realizado al PNA por el gobierno Santos, se implementaron actividades principalmente en el sector minero-energético. Así mismo, se fortaleció la aplicación de normas «voluntarias» que las empresas han empezado a utilizar para reportar sus actividades en relación con los derechos humanos. Entre ellas está el fortalecimiento de las denominadas Guías Colombia (Consejería DD. HH., 2018), sin embargo, tal y como lo manifestó la Consejería Presidencial, «no existe una línea base de indicadores que muestren los avances precisos antes de la entrada en vigor del Plan y la actualidad, por cuanto la misma no se conformó» (p. 64).

En este sentido, si bien el anterior gobierno intentó estar al día con los requerimientos internacionales para promocionar el cumplimiento de los derechos humanos y, en cierto sentido, se avanzó en la protección por parte del Estado de estos derechos, no puede decirse que en la actualidad se cumplan a cabalidad, más cuando el asesinato a líderes sociales va en aumento; situación que no solo preocupa a los organismos nacionales, sino también a la comunidad internacional.

En este momento no hay la certeza de qué pasará con este tema en el gobierno actual. Esto debido a que el PNA (y todos los PNA) tiene una vigencia de 3 años y el Plan actual se cumplió en diciembre del 2018. En tanto, el representante de la Oficina de Derechos Humanos de la ONU ha expresado algunas recomendaciones para la actualización del PNA, en las que se hace referencia a la importancia de garantizar la continuidad de lo avanzado en este tema y se sugiere incluir un balance de la legislación nacional aplicable a temas empresariales para identificar algunos vacíos en normas nacionales sobre los estándares internacionales. En caso de que se identifiquen estos vacíos, se recomienda 
ejecutar adecuaciones a las leyes, promover diálogo social intercultural entre los pueblos étnicos y las empresas, y garantizar la participación de las comunidades locales, entre otras.

A continuación, procederemos a analizar algunos ejemplos de las relaciones entre empresas y derechos humanos que, pese a que se dan en diferentes zonas, adquieren características similares. Estos puntos en común deben tenerse en cuenta para aplicar una metodología de enfoque de derechos humanos.

\section{Casos y características de derechos humanos y empresa en Colombia}

Las empresas representan un motor importante en la economía del país y su operación tendrá una relación política según el espacio territorial donde se encuentren. Por ello debe tenerse en cuenta que existe una descentralización política y administrativa con autonomía de las entidades territoriales. Aún con estas características, existen amplias deficiencias y ausencias del Estado en algunos territorios.

Así mismo, Colombia se define como un Estado multicultural, esto puede entenderse como el reconocimiento de la existencia de varios grupos poblacionales étnicos y no étnicos. Sin embargo, esta definición no es suficiente, en el entendido que en varios territorios suelen convivir diversas culturas, a lo que denominamos como interculturales. La ausencia de reconocimiento de esta característica ha generado amplios conflictos entre las diferentes poblaciones. Teniendo en cuenta que lo que existe en la actualidad es una diferenciación entre culturas y el reconocimiento de su existencia. Con este marco, en un territorio pueden existir indígenas, campesinos y afrodescendientes y, en algunos municipios, empresas. En casos como estos, la actuación y el relacionamiento de las empresas no puede ser el mismo, debe, entonces, comprenderse la diferencia que existe entre las culturas y su forma de convivir en el territorio. 
A continuación, expondremos dos casos de diferentes regiones de Colombia donde se observan algunas de las características mencionadas de este Estado; su interculturalidad, su multiculturalidad y la descentralización. Son dos regiones geográficamente alejadas una de la otra, pero que, en últimas, tienen rasgos en común que permite pensar en una aproximación sobre el tema de empresa y derechos humanos. Además, debe tenerse en cuenta que las actividades empresariales que allí se abordan fueron priorizadas para desarrollar el Plan Nacional de Acción, agro-industria e infraestructura.

\section{El caso de las empresas de la palma de aceite en María La Baja²}

El aceite de palma es un producto presente en gran cantidad de alimentos procesados, artículos de aseo personal, de limpieza, entre otros, gracias a su versatilidad y precio. Al mismo tiempo, recientemente su cultivo ha estado recibiendo críticas negativas a escala internacional, debido a que su producción ha implicado deforestación de bosques alrededor del mundo ${ }^{3}$.

En Colombia, la palma de aceite se impulsa como un cultivo que puede generar progreso en las regiones donde es sembrado. Existen diversas políticas públicas que promueven su desarrollo. En el municipio de María La Baja, ubicando en la costa norte de Colombia y parte de la subregión de Montes de María, el cultivo de palma de aceite se da junto al cultivo de alimentos para la subsistencia de las comunidades. En este municipio sus habitantes enfrentan dificultades como el acceso al agua y a la tierra, además de las consecuencias del conflicto armado.

2 En este municipio de categoría 6, según la Ley 617 del 2000, la cobertura de acueducto es del $63.7 \%$ y la de alcantarillado del $49.2 \%$. Con una población de 49138 habitantes, el 97.10 \% se identifica como población negra, mulata o afrocolombiana (DNP, 2017).

3 «El equivalente a 300 campos de fútbol de la selva tropical se destruye cada hora para dar paso a las plantaciones de palma aceitera». Veáse Mosbergen, Domique para Huffpost: We Need To Talk About Palm Oil, en https://bit.ly/2P00YiD. También puede consultarse https://bit.ly/30jNhMM, https://bit.ly/2L5dPej y https://bit.ly/2Cfke4h. 
En los años noventa, la llegada de la agroindustria de la palma de aceite coincide con el auge paramilitar y una crisis en la producción de arroz riego, el principal cultivo del municipio en esa época (Herrera y Cumplido, 2015). Para 1998 la llegada de la agroindustria a María La Baja se inserta en un periodo de crisis, cuando es especialmente vulnerable la agricultura familiar, la cual era el medio de subsistencia de las comunidades de la región. Al instalarse el negocio de la palma de aceite como un esquema de alianza productiva, se vincularon pequeños y medianos productores. De este modo, «las hectáreas sembradas de Palma de aceite en este municipio han crecido en un $830 \%$ entre los años 2001 y 2010, mientras que la tasa de crecimiento a nivel nacional para el mismo periodo fue de 141\%» (Herrera y Cumplido, 2015, p. 23). Una de las consecuencias fue que la Tasa de Sustitución de Cultivos Agrícolas por Palma de Aceite fue de 0.57 ha de cultivos de alimentos, sustituidos por cada hectárea sembrada en palma de aceite (Herrera y Cumplido, 2015, p. 75), ofreciendo empleo permanente a solo el $11.8 \%$ de la población económicamente activa. Además, la palma de aceite es un cultivo de tardío crecimiento y requiere altos costos de establecimiento y mantenimiento; los cultivos tardan unos 10 años para su estabilización, lo que es un periodo muy largo para productores pequeños, con poca tierra y pocas alternativas económicas (Álvarez, 2009).

Por otro lado, la violencia paramilitar generó más de 200000 casos de desplazamiento forzado, desapariciones, masacres, abusos sexuales, entre otros. Otra consecuencia fue el despojo de más de 80000 hectáreas de tierra entre 1997 y 2007 (De los Ríos, Becerra y Oyaga, 2012, como se cita en Ojeda, et al., 2015). Después, estas propiedades fueron tituladas y comercializadas, alimentando el latifundio de la creciente agroindustria: «El despojo en la región se caracterizó por la combinación efectiva de estrategias coercitivas y jurídicas, a través del rifle y el título» (Grajales, 2011, p. 771).

En la actualidad, esta agroindustria en Colombia es inmensamente exitosa, siendo el primer productor de aceite de palma en América ${ }^{4}$. El

4 «Colombia es el cuarto productor de aceite de palma del mundo y el primero en América». Véase Agronegocios, https://bit.ly/2sCQFmO 
contraste entre el éxito del negocio y las consecuencias que tiene sobre las comunidades de la región resalta aún más si se menciona la gran inversión realizada por el Estado para favorecer la industria palmera, a través de leyes, decretos y programas ${ }^{5}$. Como explica Rendón (2016), en 1999 comienza el apoyo al modelo de alianza productiva cuando Carlos Murgas fue ministro de agricultura, y quien además es el dueño del grupo Oleflores, uno de los principales actores de la alianza productiva de la palma de aceite. En varias ocasiones el apoyo contó no solo con recursos públicos, sino también con financiación del Banco Mundial a través del Estado.

En este punto es importante resaltar que el suelo de María La Baja es especialmente fértil ${ }^{6}$. El municipio era reconocido como la despensa agrícola de Montes de María. Sin embargo, tras el cultivo de palma los

5 Rendón (2016) explica:

Los estímulos del Estado al sector se han enfocado en: (1) facilitar el acceso a capital financiero, (2) reducir los costos de las operaciones y aportar al atractivo de los mercados, y (3) promover la autogestión del sector. (...) Primero, los estímulos de acceso a capital financiero se han realizado a través de instrumentos generados para el apoyo de pequeños y medianos productores, y en la actualidad se han adecuado para el apoyo al gran productor, como en el caso del Incentivo de Capitalización Rural (ICR) que en el Plan Colombia Siembra se amplió para dar cobertura a este tipo de productor (...) Un segundo tipo de medidas conducentes a incentivar el sector, están dirigidas a generar ambientes de excepción tributaria y arancelaria, para reducir los costos de producción y hacer más atractivos los mercados. La exención a la renta se inició en el año 2003 (ley 818). Colombia Siembra es un proyecto del Estado colombiano que pretende ampliar el área de cultivos agrícolas en el país en un millón de hectáreas más (...) Por último, el fomento a la autogestión del sector se ha realizado a partir de regulaciones de su organización colectiva, con la creación del Fondo del Fomento Palmero (ley 138 de 1994) sostenido por los mismos productores, y la incorporación de actores del sector a espacios de determinación de política pública, y a los programas de investigación, formación y desarrollo tecnológico y logístico. (L. 811/2003, Conpes 3477 de 2007 y Conpes 3510 de 2008)

6 Según el informe del perfil productivo del municipio elaborado por el PNUD, la tierra de María la Baja es una de las tierras más fértiles de Colombia. Ver página 14: https://bit.ly/2zh3rut. 
suelos fértiles escasearon ${ }^{7}$, lo que dificultó la siembra de productos de pancoger. Esto pone en riesgo la soberanía alimentaria y acentúa la alta concentración de tierra.

Además, este municipio posee dos distritos de riego que no garantizan el acceso del agua a las comunidades de la zona ${ }^{8}$. Así mismo, una revisión de la estructura de la propiedad alrededor de los distritos de riego permite apreciar que se trata de latifundios y que no son pequeños o medianos productores los que se benefician de los mismos (Duarte, 2016). Reportes que abordan las problemáticas para el acceso al agua potable en María La Baja revelan cómo la contaminación generada por el cultivo de palma no permite el goce del derecho al agua a las comunidades, e incluso genera enfermedades entre los habitantes, «especialmente de las mujeres, encargadas en su mayoría de las labores domésticas y de la alimentación de las familias» (Corporación Desarrollo Solidario, 2018).

A lo anterior debe sumarse que María La Baja no solo experimentó la violencia del auge paramilitar, los despojos de tierra y desplazamientos forzados, además, es un municipio olvidado por el Estado en otros aspectos. Apenas desde el 2016 este municipio tiene servicio de alcantarillado y tan solo en 2018 fue remodelado su acueducto. Aún así, en junio de 2018 se interpuso una acción popular en contra del municipio de María La Baja, el departamento de Bolívar y los ministerios de la Protección Social y el Medio Ambiente, exigiendo el acceso a agua potable. Según las comunidades, "por sus desechos tóxicos, los cultivos de palma han ocasionado una alta contaminación de los canales y represas de donde sale "el agua para uso doméstico y comestible de las comunidades”»

7 «Se está desperdiciando tierra fértil que podría estar utilizando en cultivos transitorios; ya que la palma africana no necesita tierras fértiles para su producción.» Véase Moreno, Yenni Carolina, Cambio de la cobertura de la tierra en María la Baja, Bolivar, entre 1984 y 2015 por proceso de despojo de tierras, p. 81.

8 «Hoy en día las y los pequeños agricultores no cuentan con condiciones para acceder a esta agua». Véase Corporación Desarrollo Solidario: El agua: fortuna y desgracia para los habitantes de María la Baja, en https://bit.ly/31IMsx8. 
(Corporación Desarrollo Solidario, 2018). Esto contrasta con todo el apoyo por parte del Estado a la agroindustria de la palma de aceite. Así como la participación de organizaciones como Fedepalma en Planes de ordenamiento territorial, municipal y departamental, planes de desarrollo municipal y departamental, planes de ordenación y manejo de cuencas hidrográficas, el fondo de ciencia, tecnología e innovación del sistema general de regalías y los proyectos que se desprendan de los planes de desarrollo y otros escenarios ${ }^{9}$. A esto se suma el apoyo que ha recibido la industria de la palma por parte del Estado. El apoyo político y la capacidad de incidencia que puede apreciarse en este ámbito es muy significativo para las empresas de la industria de la palma, lo cual contrasta con el apoyo que reciben las comunidades.

Frente a la responsabilidad social de las empresas con el territorio, el grupo Oleoflores y su fundación Fundemaría se enfocan en apoyar proyectos productivos locales, educación, cultura ciudadana, valores, generación de empleo y autoconstrucción de vereda rural en Montes de María, subregión donde se encuentra María La Baja (Grupo Oleoflores, 2015). Aunque es indiscutible que estos esfuerzos son válidos y apuntan a la transformación regional, no responden a las principales demandas de las comunidades generadas por la presencia del modelo productivo de la palma en el territorio, que como ya se ha visto, incluyen el acceso a la tierra y al agua potable. Puede afirmarse que son esfuerzos de responsabilidad social que buscan crear un impacto positivo, pero no logran mitigar las consecuencias de la agroindustria de la palma sobre las comunidades en el territorio.

Así mismo, debe tenerse en cuenta que María La Baja es un territorio multicultural con presencia de población indígena y afrocolombiana; hay dos (2) cabildos indígenas y diez (10) consejos comunitarios. A lo largo de los años las comunidades han creado fuertes procesos

9 Esta información está disponible en un organigrama de Fedepalma disponible en línea: https://bit.ly/31PCGJN. 
organizativos para el reclamo de sus derechos. Por ejemplo, la Mesa Permanente por el Derecho al agua potable; varias ONG y organizaciones sociales y académicas actúan como facilitadores de estos procesos y se encargan de reportar abusos en contra de las comunidades ${ }^{10}$.

\section{El caso de las empresas portuarias en Buenaventura ${ }^{11}$}

La historia de Buenaventura fue marcada por el cambio que trajo consigo el paso de ser una pequeña ciudad junto al mar con una población principalmente dedicada a la agricultura y la pesca, a ser la ciudad puerto más importante del país. Desde 1944 Buenaventura alcanzó volúmenes de importaciones y exportaciones similares a los demás puertos colombianos y desde entonces ha seguido en alza, convirtiéndose en el puerto del país con la mejor posición geoestratégica, con salida al canal de Panamá y siendo el más próximo del continente americano de los mercados asiáticos.

En 1961, la empresa Colpuertos entró como operadora de los puertos en Colombia, una empresa pública que influyó directamente en la relación política, social y laboral, generando empleo a más de cinco mil

10 Se incluye Sembrando paz, Fundación Red Desarrollo y Paz, Corporación Desarrollo Solidario, Red ASVIDAS de los Montes de María, Fundación para el desarrollo social de María La Baja, entre otras.

11 Es un municipio ubicado en la subregión cultural del pacífico sur colombiano. Comprende desde el río San Juan hasta el río Mataje en la frontera con Ecuador y desde la cordillera occidental hasta la línea costera con el océano pacífico. Es el municipio más extenso del departamento del Valle del Cauca con $6297 \mathrm{~km}^{2}$, cifra equivalente al $29.7 \%$ del departamento. La zona rural del municipio está conformada administrativamente por 19 corregimientos y 31 consejos comunitarios de comunidades negras (con titulación constituida en cumplimiento de la Ley 70 de 1993 y del Decreto 1745 de 1995) y por 9 resguardos indígenas pertenecientes a los grupos étnicos waunan, embera, eperara, siapiadora, nasa y embera chamí.

De acuerdo con el DANE (Departamento Administrativo Nacional de Estadística), para el 2011 el municipio de Buenaventura tenía 369753 habitantes, de los cuales 51 \% eran mujeres (188 574) y 49 \% eran hombres (181 179) Para el mismo año 2011, los grupos étnicos se distribuían en: $73 \%$ afrocolombiano, $26 \%$ mestizo, $0.8 \%$ raizal y $0.2 \%$ indígena (CNMH, 2015, pp. 29, 31, 33 y 35). 
trabajadores nativos de la región. La empresa fortaleció el surgimiento de élites políticas locales y la construcción de una fuerte organización sindical (CNMH, 2015, p. 46). Para 1993, y mediante la Ley $1^{\text {ra }}$ del mismo año, fue dado en concesión el manejo de los puertos, con el argumento de que la administración pública había sido ineficiente y contaba con un sobrecosto en mano de obra, lo que desde entonces ha traído para la población fuertes cambios en su dinámica económica y social.

De acuerdo con el Centro Nacional de Memoria Histórica (2015), a pesar de que Buenaventura es la ciudad puerto más importante en el pacífico colombiano, es una ciudad con un alto índice de inequidad, una profunda desigualdad social y pobreza. En medio de la riqueza económica de los puertos es el tercer municipio más pobre del Valle del Cauca. En Buenaventura quienes poseen más riqueza están 66 veces por encima de aquellos más pobres, lo que evidencia una ciudad con una brecha muy amplia de desigualdad. Además, según un reporte de Semana (2017), se resalta el deterioro de sus vías; las construcciones primarias; la falta de agua potable las 24 horas del día; no cuentan con un hospital de primer nivel; y los índices de pobreza son muy altos. El Departamento Nacional de Planeación afirma que mientras a nivel nacional el índice de pobreza multidimensional es del $49 \%$, en Buenaventura alcanza el 66 \% . Por otra parte, se calcula que el $64 \%$ de la población urbana y el 91 \% de la población rural son considerados pobres y el 9,1 \% vive en condiciones de miseria. A esto se agrega que,

[...] en Buenaventura la tasa de desempleo es del $62 \%$ y el empleo informal llega al 90.3\% según cifras también del DNP. La problemática aumenta frente a la queja de sus habitantes, ya que, pese a que el puerto mueve más de 15 millones de toneladas de carga al año, no hay trabajo para ellos. (Semana 2017, párr. 10)

En Buenaventura operan: la Sociedad Portuaria, la cual tiene el mayor porcentaje de la carga que llega al puerto con un $70 \%$; la terminal de contenedores TCBUEN; el Grupo Portuario y la compañía Compas, 
de propiedad internacional y el terminal AGUADULCE, inaugurado en el 2017, el cual es también de inversión extranjera. Todas estas sociedades han llegado al territorio con proyectos logísticos que favorecen la economía nacional al convertir a Colombia en un país competente en la comercialización marítima. Sin embargo, su centro de operaciones, Buenaventura y su población no ve esta riqueza, pese a las millonarias cifras que mueve su puerto. Por el contrario, es poco el desarrollo de la ciudad y es más lo que padece su población que la responsabilidad social que asumen estas empresas.

Existen ejemplos de la inversión social hecha por privados, es el caso de la Fundación Sociedad Puerto Industrial Aguadulce Buenaventura. Una fundación que fue creada en el 2012 para promover y apoyar proyectos sociales en el puerto y así proporcionar beneficios a la población bonaverense. Se trata de una apuesta de los privados en sus esfuerzos de responsabilidad social. Sin embargo, no terminan alcanzando su objetivo y, por el contrario, pueden afectar el tejido social de las comunidades debido a que el beneficio no es colectivo y, por el contrario, está concentrado en aquellos más cercanos a la empresa, sobre todo, en lo que refiere a las oportunidades laborales. La privatización de los puertos trajo como consecuencia la no integración de los habitantes de Buenaventura a la cadena de valor de las empresas ${ }^{12}$. Los locales no suelen ser contratados, y por ende no se benefician de la actividad económica del puerto.

Buenaventura es ejemplo de cómo la coexistencia entre grandes proyectos de infraestructura y las comunidades no se traduce en desarrollo económico, por el contrario, es causa de pobreza y desigualdad; una ciudad de contrastes entre la riqueza del puerto y la miseria con la que muchos pobladores viven. Esto es paradójico, pues a lo largo del tiempo, la llegada de empresas al territorio nacional ha significado una

12 Tal como se menciona en la publicación del Centro Nacional de Memoria Histórica, Buenaventura, un puerto sin comunidad, disponible en https://bit.ly/2v4woq3. 
aproximación a lo que comúnmente se traduce en desarrollo, y con políticas estatales se ha abierto el camino para recibir a las grandes empresas nacionales o extranjeras, pero el descuido en la parte social y la premisa de crecer económicamente generan este tipo de situaciones contrastantes, en donde aquellos con potencial económico reciben más beneficios que los habitantes del territorio. Así mismo, los proyectos de infraestructura que trajo consigo la privatización de los puertos han terminado por desplazar a los habitantes de sus territorios y de las zonas de donde obtienen mayor fuente de recursos para sobrevivir. A lo anterior se suma el hecho de no obtener a cambio nuevas oportunidades. La población de Buenaventura ha visto la destrucción de sus viviendas y la reubicación de sus familias en entornos contaminados, cercanos a las fuentes de ruido y al desorden que producen los grandes contenedores cargados de mercancías. En definitiva, no ha gozado de unos mínimos en condiciones de vida.

Debido a toda esta gran crisis que ha atravesado por décadas Buenaventura, se organizó una movilización que tuvo lugar en mayo del 2017, cuando la población bonaverense se organizó con base en lo «cívico». De esta manera, desarrolló durante 22 días un paro que congeló el comercio, la venta de gasolina y el transporte terrestre de mercancías, entre otras. Este paro tuvo como objetivo exigirle al Gobierno nacional una inversión económica que permitiera el acceso al agua potable para el 100 \% de la población; la reapertura del hospital distrital de nivel dos, el cual funciona únicamente como puesto de salud; educación de calidad y empleo. Entre tantas, los líderes sociales del movimiento paro cívico lograron sentar a la mesa a diferentes ministros y a la gobernadora del Valle, y durante los 22 días de paro negociaron la forma como acordarían subsanar las graves problemáticas sociales y económicas de Buenaventura.

Actualmente, el movimiento del paro cívico ejerce un gran poder social y actúa como principal vocero de la población bonaverense, brindando una oportunidad para llegar a diferentes esferas políticas y sociales con 
las necesidades que se presentan en el territorio. La organización social parece ser la mejor herramienta para solicitar la mirada del Estado que durante tantos años ha sido esquiva hacia el puerto sobre el Pacífico.

\section{Aspectos comunes en estos territorios}

En estos dos casos se observan puntos en común sobre los territorios y la forma como las empresas han acudido a posicionarse no solo con un poder económico, sino también político. Es importante resaltar, también, que existen denuncias en las dos zonas, en las que existen empresas o personas diferentes que las representan, tanto de aceite de palma ${ }^{13}$, como de los puertos ${ }^{14}$. Se trata de personas y/o empresas que se han involucrado en despojo de tierras e incluso en muertes de líderes sociales, situaciones que, sin lugar a duda, afectan el derecho a la vida. Por otra parte, se han denunciado cambios ambientales que afectan no solo el derecho a un ambiente sano, sino también a la vida misma.

Así mismo, se considera importante realizar un análisis de algunas características que tienen en común estos territorios con el objetivo de dar paso a una aproximación metodológica que contribuya a evitar que estas situaciones se presenten en el país y garantizar plenamente los derechos humanos por parte de las empresas que operan en Colombia.

13 Por ejemplo, el caso de la Hacienda el Cucal, que «es reclamada por víctimas del conflicto armado. Su proceso se ve entorpecido, dicen, porque la palma cultivada allí es explotada por Oleoflores, una empresa del exministro de Agricultura Carlos Murgas Guerrero». Véase Rutas del Conflicto, en https://bit.ly/2YWeusc. También se menciona en La tierra en disputa del Centro Nacional de Memoria Histórica, disponible en https://bit.ly/2HadiGM.

14 Líderes sociales que han denunciado la expansión de los puertos han sido asesinados, situación que se difumina con la amplia violencia que existe en esta zona. Véase por ejemplo el Comunicado internacional de denuncia del asesinato de líder social de Buenaventura, Temístocles Machado, disponible en https://bit.ly/2Hadgi8. 


\section{Coexistencia de una actividad económica exitosa en un entorno socioeconómico donde la presencia de esta actividad no ha generado mayor desarrollo económico local}

La presencia de un actor económico que genera inmensa riqueza no se traduce en desarrollo local en el territorio. Por desarrollo económico local puede entenderse el «Proceso de crecimiento y cambio estructural que, mediante la utilización del potencial de desarrollo existente en el territorio, conduce a elevar el bienestar de la población» (VázquezBarquero, 2001, p. 21, como se cita en Rendón, 2016).

En este sentido, además de la ausencia de desarrollo económico local que se observa en María La Baja y Buenaventura, es importante resaltar cómo chocan diferentes perspectivas de lo que se entiende como desarrollo. Las prácticas económicas de las comunidades en su territorio se vieron truncadas con la llegada del actor económico, más aún, las prácticas han contribuido a la vulneración de derechos económicos y sociales. Es decir, no hubo una inserción de la actividad económica en las dinámicas territoriales previas a su llegada, de manera que permitieran una articulación en pro de un crecimiento paralelo entorno-empresa.

Las aspiraciones de desarrollo de las comunidades se tratan principalmente de la garantía de sus derechos económicos, sociales y culturales, como la subsistencia, el goce de los recursos naturales, el goce de su territorio, la vivienda, así como el desarrollo de su cultura y prácticas ancestrales que incluyen su forma de vida económica. Esta visión del desarrollo, al convivir en el mismo territorio y compartir los mismos recursos naturales, choca con el desarrollo traído por modelos agroindustriales e industriales.

\section{Beneplácito estatal}

El Estado colombiano tiene una presencia débil en los lugares rurales, en especial en aquellos más alejados de las grandes ciudades. Sin embargo, pareciera que no es solo débil, sino que, además, a través de sus acciones 
favorece a las empresas y omite beneficios a las comunidades. En Colombia es frecuente hablar de aquiescencia estatal, ya que, por un lado, el Estado no garantiza derechos para los habitantes, pero sí facilita la inserción en el territorio de algunas malas prácticas desempeñadas por empresas que vulneran los derechos humanos, ya sean nacionales o internacionales, a pesar de que esto vaya en detrimento de la propia cultura o de las comunidades.

En María La Baja y Buenaventura el Estado tiene una larga historia de no haber sido garante de los derechos humanos. Esto ha generado manifestaciones y paros para exigir a las autoridades que asuman la responsabilidad y cumplan con la promoción y protección de los derechos. Pese a las necesidades vitales que tienen, la inversión que se realiza no es eficiente, y gran parte de esta no se enfoca en beneficiar a la población en general, sino que se encuentra, muchas veces, orientada en favorecer los intereses de los sectores empresariales.

\section{Detrimento de las dinámicas socioculturales de las comunidades en el entorno donde se desarrolla la actividad económica}

En ambos territorios, el proceso de instalación de la actividad económica trajo consigo un cambio en los procesos sociales y las dinámicas organizativas de las comunidades afectadas. Tradiciones y hasta métodos de subsistencia cambiaron radicalmente y se vieron forzados a integrarse a este nuevo modelo de desarrollo, intentos que en su mayoría no fueron exitosos.

Es difícil llevar a la práctica la lógica de desarrollo consistente en que el crecimiento debe darse mediante la utilización del capital existente en los territorios y elevar con este el bienestar de la población, ya que la lógica propia de cada comunidad étnica varía según su creencia, cosmovisión y localización; lo cual no es tenido en cuenta por las empresas que llegan a los territorios y, por el contrario, se cambia el estilo de vida de las comunidades y se convierte en un estilo de vida que se ajusta al referente de la empresa. Es decir, no hay respeto por 
la visión del territorio entendida por sus propios habitantes, se afirma, por el contrario, la intromisión de la lógica industrial que, en ocasiones, fractura la identidad comunitaria, deteriora el tejido social y empobrece aún más la sociedad, esto, a su vez, puede repercutir en vulneraciones a derechos colectivos.

\section{Empoderamiento de terceros alrededor de la garantía de derechos económicos, sociales y culturales}

En ambos casos, las empresas, fundaciones y ONG han adquirido un rol que pertenece, en realidad, al Estado en cuanto a la garantía de derechos económicos y sociales. Grandes empresas se transforman en actores políticos, al participar en planes de desarrollo y en otros espacios comunitarios de participación, como los consejos regionales de desarrollo. Las comunidades pierden entonces poder para incidir en estos espacios, que precisamente están diseñados para una toma colectiva de decisiones sobre el desarrollo de los habitantes del territorio. Esto, en especial si la empresa tiene la posibilidad de moldear políticas públicas o programas a su favor.

Por otro lado, las organizaciones que realizan intervenciones sociales también han afectado las dinámicas de las comunidades, debilitando el tejido social en algunos casos. Por ejemplo, al ser excesivamente asistencialistas vulneran la autonomía de las comunidades. En entrevistas realizadas a líderes sociales por parte del IEI en el 2016, en los Montes de María, se evidenció la manera en que las fundaciones, al financiar espacios de encuentro de líderes que se daban incluso antes de que la misma estuviera presente, le restaban espontaneidad al espacio, perdiendo su valor per se.

Adicionalmente, se identifican programas de Responsabilidad Social por parte de las Empresas. Pero, un elemento en común es que estos esfuerzos no responden a mitigar el impacto directo de la empresa en el territorio, dado que se enfocan en suplir otro tipo de demandas de la comunidad que, idealmente, también deberían ser cumplidas por el Estado. 
Buenaventura se enmarca en la misma lógica; el grueso de la población evidencia la pobreza y desolación que padecen, y pese a existir fundaciones y acciones por parte de organizaciones privadas, este proceso se da de manera selectiva: solo aquellos más próximos pueden gozar de los beneficios que ofrecen en temas de salud, educación, medioambiente, entre otras. Es necesario, por tanto, la articulación con el Estado para un desarrollo colectivo y genuino de la población.

\section{Empoderamiento del rol de las organizaciones sociales}

En ambos casos las organizaciones sociales (consejos comunitarios, mesas de interlocución, entre otras) han adquirido un rol clave en la defensa y el reclamo de los derechos de las comunidades. Los liderazgos sociales se han transformado en posiciones de lucha, se enfrentan amenazas, y a pesar de la existencia de espacios de interlocución, se reclama que no están siendo escuchados suficientemente por el Estado. Por ello, se toman medidas para reclamar sus derechos, como el paro cívico de Buenaventura o movilizaciones campesinas en María la Baja para la reivindicación de sus derechos (El Universal, 2018).

De manera general y en los casos previamente expuestos, es evidente cómo al no haber una presencia institucional robusta por parte del Estado, la articulación y movilización social visibilizan las necesidades de los territorios, a veces siendo la única herramienta con la cual cuentan las comunidades para alzar la voz de protesta y ser escuchadas.

\section{Metodología para que las empresas desarrollen el cumplimiento de los derechos humanos}

Tomando en cuenta cómo va Colombia en la garantía de los derechos humanos y su relación con las acciones de las empresas, y las características de los casos expuestos, es importante anotar que las empresas que desarrollan estas actividades (agroindustria e infraestructura) han olvidado u omitido su responsabilidad por preservar los derechos humanos. Debe resaltarse que los puntos priorizados por el PNA 
contenían la agroindustria y la infraestructura como los principales temas a desarrollar. Sin embargo, en su ejecución se ha avanzado principalmente en el tema minero, en donde se cuenta con una amplia participación de algunas empresas y declaración de políticas al interior de las mismas, ejemplo de ello es que en Valledupar las compañías Drummond, Prodeco y Cerrejón firmaron una declaración conjunta para proteger la vida de los líderes sociales de la región (FIP, 2018).

Para lograr la aplicación de los PR en Colombia no solo existe el PNA, además, se han generado internacionalmente los puntos mínimos para hacer efectivos los derechos humanos en las diferentes empresas a través de guías. Sin embargo, consideramos que, dadas las características del país, estas guías son insuficientes, y viendo el análisis de los dos casos mencionados, es importante distinguir una metodología que ayude a superar estas situaciones. Para solucionar estos inconvenientes, que no solo suceden con estas empresas, y en estos territorios, sino que los mismos son una pequeña radiografía de la generalidad en Colombia, es importante asumir el desarrollo de los derechos humanos, pero adicionando la diferenciación de territorios multiculturales e interculturales. Por ello, en este apartado y a manera de reflexión, se plantean cinco puntos comunes que deben ser abordados a través de una metodología que incluya a la comunidad donde hacen presencia las empresas.

\section{Diagnóstico}

La Guía de Evaluación y Gestión de Impactos en los Derechos Humanos (EGIDH) escrita por Désirée Abrahams y Yann Wyss (2010), incluye una etapa de preparación en la que se visualiza cómo está la empresa en materia de derechos humanos y cómo debe aplicarse la debida diligencia. Una siguiente etapa es la identificación, en la que se establecen los riesgos e impactos de la empresa en materia de derechos humanos. Por último, existe una etapa de relación, que establece cómo son las relaciones de la empresa con la comunidad. 
Consideramos que es importante unir estos dos aspectos en un punto denominado diagnóstico, el cual dará evidencias al interior y al exterior de la empresa. Este diagnóstico implica remontarse al inicio de la empresa y preguntarse cómo ha sido el relacionamiento con su entorno, así como las relaciones existentes al interior. Se incluye, además, una revisión en la cadena de valor y se registran los efectos de sus procesos sobre el entorno y los actores presentes en el territorio, si estos han generado cambios en su estilo de vida y tradiciones. Para esto, primero es necesario conocer qué características tiene la comunidad que se encuentra cerca, si se trata de una comunidad étnica, comprender cuál es su relación con el territorio, cómo conciben los recursos naturales, cuál es su jurisdicción, su relación con la tierra, cómo es la estructura de la propiedad, su historia de poblamiento, así como sus prácticas culturales. Esto incluye determinación de actores, características al interior y exterior de la empresa, establecer la historia y las dinámicas del poblamiento, así como la historia de la empresa en el territorio. Para reconocer la ancestralidad en los entornos multiculturales e interculturales es importante conocer la comunidad, preguntarse cuál es su cultura, cómo es el relacionamiento con el territorio y cómo resuelven sus conflictos.

Al respecto, es importante resaltar que el diagnóstico genera una nueva posibilidad de realizar las actividades de una forma diferente y da inicio a lo que Salmón considera

[...] un cambio en la manera de entender cómo hacer negocios: se empieza a hablar de la responsabilidad social empresarial como una manera distinta (y con sentido ético) de trabajar y generar riqueza. Es decir, se asume que es posible llevar a cabo actividades rentables y responsables a la vez, logrando con ello mejoras de carácter financiero sostenidas en relaciones de cuidado con los grupos con los que la empresa interactúa. (Salmón, Blanco y Zambrano, 2016, p. 16). 
Como puntos a seguir, sería importante:

Determinar quiénes son las personas adecuadas para realizar este diagnóstico. Es importante determinar si alguien de la empresa está en la capacidad de realizarlo o si se requiere un nuevo cargo o, en su defecto, la contratación de una entidad que pueda asumirlo, ya que durante este diagnóstico se deberán realizar diferentes entrevistas en la comunidad, las preguntas estarán encaminadas a hacer un reporte de hallazgos de lo que se observa en el entorno, con su respectivo análisis.

Al interior de la empresa: las preguntas de la entrevista estarán encaminadas a construir hallazgos que den cuenta de quiénes son las personas que se relacionan con la comunidad y cuál es la visión que tienen, como empresa, de su entorno.

Generar una línea de tiempo que dé cuenta de la historia del territorio: cuáles eran las prácticas antes de la llegada a la empresa, cuáles han sido las prácticas culturales que se han perdido a lo largo del tiempo; qué actores sociales tienen más relacionamiento con la empresa y cómo es el proceso de liderazgo que existe en el entorno. Si existió o existe conflicto armado en el territorio, se debe documentar cuáles son los hechos más relevantes que puedan generar alguna vinculación de la empresa.

Observar cuáles son los problemas de orden público y las deficiencias del Estado en el territorio.

\section{Diseño de plan de mitigación}

En este punto es importante tener en cuenta los resultados obtenidos del análisis al diagnóstico, para ello es importante tener como norte las palabras expresadas por Ruggie,

[...] Los derechos humanos no son simplemente el proverbial canario de la mina que sirve para indicar que algo no funciona, el respeto por la dignidad humana también debe y tiene que construir uno de los pilares sobre 
los que deben colmarse las lagunas de gobernanza, tanto desde el nivel local como global, y en el sector privado no menos que en el público. Para que la empresa sea más justa en relación con los derechos humanos, es preciso encontrar formas de convertir el respeto de los derechos humanos en parte integrante de la empresa, es decir, convertir este respeto en una práctica estándar empresarial. (Ruggie 2014, p. 21)

De acuerdo con el análisis de los hallazgos encontrados, saber cómo se ha intervenido en la comunidad, conocer cuáles eran sus prácticas, reconocerlas y darles valor e importancia a las mismas. Lograr concebir el desarrollo social como el principal motor del desarrollo económico, siempre preservando la dignidad humana.

En este sentido, será importante diseñar un plan encaminado a transformar las políticas empresariales y adaptarlo a la sociedad donde se encuentra. En la EGIDH podría considerarse si corresponde a la evaluación, que no es otra cosa que establecer los impactos en los derechos humanos y la mitigación, lo que se traduce en el desarrollo de los planes para mitigar las vulneraciones.

El diseño del plan debe contar con la participación de la comunidad, para que esta también pueda validar cómo se garantizan sus derechos humanos. En este sentido, Ruggie (2014) establece un término que denomina la licencia social, que no es otra cosa que la aceptación y el permiso de la comunidad para actuar, y allí se puede observar que es un hecho cierto que en muchos ámbitos donde realizan actividades las empresas identifican necesidades que podrían satisfacer para poder actuar sin problemas en su entorno. Sin embargo, ¿qué pasa cuando estas comunidades no tienen la misma visión de necesidades? Las necesidades se construyen de acuerdo con sus entornos, y por qué no decirlo, con los modelos de desarrollo que pretenden ejecutarse. Las comunidades suelen no coincidir con esa visión de desarrollo, ya que la suya es diferente: poder continuar con su vida, en su territorio, en mejores condiciones, sin que sus costumbres sean suprimidas. Es en este 
sentido que el plan se identifica con lo concebido por las comunidades, y no con una única visión de desarrollo.

\section{Ejecución del programa}

Dentro de la empresa, podría manifestarse que existe una coincidencia con lo que la EGIDH considera es la gerencia, que está principalmente al interior de la empresa para integrar la política de derechos humanos. Sin embargo, esta gerencia incide directamente en la comunidad, por ello la manifestación de la política de derechos humanos es un compromiso con su entorno. Allí se deben llevar a cabo diferentes actividades, de modo que generen un nuevo relacionamiento entre la empresa y el entorno.

Es fundamental que en las empresas se generen políticas que persuadan al Estado, de manera que este asuma su verdadero rol. Además, que se ejerzan actividades de comunicación según el entorno en donde se encuentran, esto será posible en la medida en que conozca bien a su interlocutor, de modo que sea posible generar un verdadero intercambio de saberes.

La falta de garantías de derechos fundamentales por parte del Estado es un punto en común en los casos de Buenaventura y María La Baja, y es una de las causas de la creación de relaciones de dependencia entre las empresas y las comunidades, en las que, además, el Estado se libera de su responsabilidad. La intención de este punto es transformar esta relación. Esto debe darse desde una perspectiva no filantrópica, es decir, debe tener en cuenta a la sociedad y a su entorno, de forma que puedan generarse consensos adecuados donde juntos puedan solicitar al Estado las transformaciones que requieren, con el fin de tener un entorno adecuado.

\section{Seguimiento}

En la EGIDH esta etapa es concebida como la evaluación, y se refiere al monitoreo de los impactos en derechos humanos. Nosotros concebimos 
esta etapa como una valoración de las actividades desarrolladas. Esto es, por ejemplo, aclarar cuál es la postura al interior de la empresa y la comunidad. Lo anterior teniendo en cuenta que el cumplimiento de la política de derechos humanos creada es la nueva forma de relacionarse $\mathrm{y}$, en este sentido, sus acciones estarán encaminadas a cumplirlas. Para ello se deberá hacer una valoración periódica de las transformaciones alcanzadas. No se trata simplemente de cumplir indicadores que den por sentado un avance, sino evaluar real y cualitativamente qué se ha modificado.

Por ejemplo: ¿cómo ve la comunidad a la empresa una vez implementadas las políticas?, ¿cómo se identifican esos cambios?, ¿la comunidad ha podido seguir ejerciendo sus prácticas ancestrales? Evaluar al interior de la empresa si se ha cambiado la visión que se tiene sobre la comunidad y si los procesos de comunicación y relacionamiento han incidido.

Es en esta etapa donde se genera una política a largo plazo, ya que, si por algún motivo no existen avances, la misma debe mejorar los aspectos necesarios para llevar a cabo una buena práctica de derechos humanos.

En este sentido, la necesidad de avanzar en la aplicación de una política de derechos humanos al interior de las empresas generará relaciones más cercanas con las comunidades, podría decirse, que incidirá directamente en la sostenibilidad tanto de la empresa como de su entorno, ya que algunos conflictos podrían evitarse o darse manejos adecuados a las diferencias. Por lo pronto, se continúa a la espera del nuevo Plan Nacional de Acción, con la esperanza de que se acojan las reflexiones realizadas por el representante de la ONU, para adecuar las normas existentes a los PR. Así mismo, se espera que se promueva el desarrollo de políticas al interior de las empresas para preservar los derechos humanos y el adecuado relacionamiento con su entorno.

\section{Conclusiones}

Los casos expuestos permiten concluir, a nivel general y especifico, que en Colombia se aprecia un nuevo rol de las empresas frente a los derechos 
humanos. Esto se evidencia en el amplio poder político que tiene estos agentes económicos y en su influencia sobre lo público.

En Colombia se había avanzado mucho al respecto y las empresas reunidas en diferentes gremios habían adelantado acciones para cumplir con debida diligencia la protección de los derechos humanos. Sin embargo, en la actualidad la política pública no ha tenido mayores modificaciones. Es importante que las empresas acaten y preserven los derechos humanos. En este sentido, el orden internacional y la vigilancia de la población civil están llevando a sancionar, de una u otra forma, las vulneraciones que con su actividad puedan ocasionar en contra de estos derechos.

La metodología propuesta responde a las dificultades halladas en la relación entre las empresas y las comunidades frente a la vulneración de derechos humanos. Poder desarrollar las actividades empresariales acorde con la metodología presentada puede generar un relacionamiento más armónico con las comunidades y el entorno donde se encuentran y, con ello, evitar y prevenir la vulneración de los derechos humanos. Sin embargo, el éxito de esta metodología está ligado no solo a que la empresa tenga la voluntad de hacer cumplir estos derechos, sino también a que el Estado genere incentivos para su cumplimiento.

\section{Referencias}

Abrahams, Désirée y Wyss, Yann (2010). Guía de Evaluación y Gestión de los Impactos de los Derechos Humanos (EGIDH). (International Business Leaders Forum [IBLF] y International Finance Corporation [IFC]. Recuperado de www.ifc.org/hriam

Cantú, Humberto (2017). Planes de acción nacional sobre empresas y derechos humanos: sobre la instrumentalización del derecho internacional en el ámbito interno. Anuario Mexicano de Derecho Internacional, XVII, 113144. Recuperado de https://bit.ly/2Z95j2z

Centro Nacional de Memoria Histórica [CNMH] (2015). Buenaventura: un puerto sin comunidad. Bogotá: CNMH. 
Consejería Presidencial para los Derechos Humanos (2018). Segundo informe de seguimiento del Plan Nacional de Acción de Derechos Humanos y Empresas 2018. Bogotá. Recuperado de https://n9.cl/mdsb

Corporación Desarrollo Solidario (2018) ¿Por qué el agua de Marialabaja enferma a las mujeres? Recuperado de https://bit.ly/2NYo1qq

Duarte, Carlos (2016). Desencuentros Territoriales 2 - Los conflictos territoriales en la Altillanura, Putumayo y Montes de María. Instituto de Estudios Interculturales. Recuperado de https://n9.cl/bmgd

De la Cuesta, Martha y Valor, Carmen (2003) Responsabilidad social de la empresa. Concepto, medición y desarrollo en España. Boletín económico de ICE, Información Comercial Española, ISSN 0214-8307, Nº 2755, 2003, pags. 7-20 Recuperado de https://n9.cl/9hdh

De los Ríos, Edwin, Carmen Andrea Becerra y Fabián Enrique Oyaga (2012) Montes de María. Entre la consolidación del territorio y el acaparamiento de tierras. Bogotá: ILSA. Disponible en https://n9.cl/2eylq Citado en Ojeda, Diana, Petzl, Jennifer, Quiroga, Catalina, Rodríguez, Ana Catalina, \& Rojas, Juan Guillermo. (2015). Paisajes del despojo cotidiano: acaparamiento de tierra y agua en Montes de María, Colombia. Revista de Estudios Sociales, (54), 107-119. Recuperado de https://dx.doi.org/10.7440/res54.2015.08

Departamento Administrativo Nacional de Estadística [DANE] (2017). Departamento de Planeación Nacional [video]. Recuperado de https://terridata. dnp.gov.co/\#/perfiles

Fundación Ideas para la Paz (2018) FIP firma declaración para la protección y respeto de los líderes y defensores de Derechos Humanos. Página web de la Fundación, sección Noticias FIP. Recuperado de https://n9.cl/igzh

Grajales, Jacobo (2011). The Rifle and the Title: Paramilitary Violence, Land Grab and Land Control in Colombia. Journal of Peasant Studies, 38, 771792.

Grupo Oleoflores (s.f.). Grupo Oleoflores, fundaciones. Recuperado de http:// www.oleoflores.com/fundaciones/fundemaria

Herrera, Gustavo y Cumplido, Vicente (2015). Implicaciones de la palma de aceite en la estructura productiva agrícola y la seguridad alimentaria del municipio de María la Baja - Bolívar [Tesis de maestría]. Universidad Tecnológica de Bolívar. Recuperado de https://bit.ly/2TJJ7Lw 
IEI. (2016). Instituto de Estudios Interculturales. Lectura territorial Montes de María.(documento inédito)

Martínez, Magary (2018). ¿Por qué el agua de María La Baja enferma a las mujeres? Corporación Desarrollo Solidario. Recuperado de https://bit. ly/2uqoxob

ONU Derechos Humanos [ONU DD. HH.] (2018). Balance y recomendaciones para la actualización del Plan Nacional de Acción en Derechos Humanos y Empresa. ONU DD. HH.-Colombia. Recuperado de https://bit.ly/2Z8xnHz

OCDE (2013), Líneas Directrices de la OCDE para Empresas Multinacionales, OECD Recuperado de https://bit.ly/36P4mlo

Ojeda, Diana; Petzl, Jennifer; Quiroga, Catalina; Rodríguez, Ana Catalina; Guillermo Rojas, Juan (s.f.). Paisajes del despojo cotidiano: acaparamiento de tierra y agua en Montes de María, Colombia. Colombia. Recuperado de https://bit.ly/33JFYQz

Porras, Yessica (2018). Campesinos de Bolívar marcharon desde Marialabaja a Turbaco pidiendo garantías. El Universal. Recuperado de https://bit. ly/2P2tSyr

Prado, Mario (2018). La militarización de Buenaventura. El Espectador. Recuperado de https://bit.ly/2SFMH81

Revista Semana (2017). Las seis deudas históricas por las que protestan en Buenaventura. Revista Semana. Recuperado de https://bit.ly/2LQnVRV

Rendón, Felipe (2016). Agroindustria y desarrollo en un territorio en posguerra en Colombia. El caso de la palma de aceite en María la Baja [Tesis de maestría]. El Colegio de la Frontera Norte. Recuperada de: https://bit. ly/2TGJzKx

Ruggie, John (2014). ¿Solamente negocios? Barcelona: Icaria.

(2018). ¿Jerarquía o ecosistema? La regulación de los riesgos relativos a los derechos humanos provenientes de las empresas multinacionales. En Rodríguez, César (ed.), Empresas y derechos humanos en el siglo XXI (p. 320). Buenos Aires: Siglo Veintiuno. 
Salmón, Elizabeth; Blanco, Cristian y Zambrano, Gustavo (2016). La progresiva incorporación de las empresas en la lógica de los derechos humanos. Lima: Universidad Católica del Perú.

Vivas, Julián (2017). El drama por el agua que encendió el paró en Buenaventura. El Tiempo. Recuperado de https://bit.ly/2NgBGtR 